the reported drop of 50 per cent in the proportion of US students choosing to graduate in science between 1966 and 1988. Yet whether this has any more bearing on the progress of the Great Society than has the "functional illiteracy" of 25 per cent of American youth, or indeed expresident Reagan's arresting opinion that trees cause pollution, cannot be determined. Such (not wholly disinterested) bodies as the AAAS and the NSF have, it is true, asserted otherwise, and foresee a desperate dearth of science graduates in the next decade; but perhaps the drift from science simply reflects the level of opportunity, not to mention emolument, available to young scientists today. In Britain such must undoubtedly be the case. In the United States, students do at any rate have the chance of dropping out of science, having at least been exposed to a little of it, and into the humanities. In Britain, if they get to university at all, a change of subject is seldom a realistic option. If British students choose to throw in the diaper and seek their future in accountancy, it may after all then be not because they are dumb but because they are smart.

Walter Gratzer is in the MRC Cell Biophysics Unit, 26-29 Drury Lane, London WC2B 5RL, UK.

\section{Starting matter}

\section{David W. Hughes}

The Origin of Comets. By M. E. Bailey, S. V. M. Clube and W. M. Napier. Pergamon: 1990. Pp. 577. Hbk £39, pbk£19.59.

PEOPLE studying the origin of comets are confronted with an extremely difficult task. Comets are not like stars - we cannot see regions of space in which comet formation is taking place at the present time. The strong possibility that cometary formation took place 4,570 million years ago, at the dawn of the Solar System, helps little too. Comets are not like asteroids - they do not fall to Earth - so our knowledge of their composition is rudimentary. We know that the cometary nucleus is made up of snow and dust, and that most of the snow is $\mathrm{H}_{2} \mathrm{O}$ and the dust is similar to primitive carbonaceous chondritic meteorites. But we have little idea as to the minor constituents, or whether the composition is the same throughout the nucleus, or whether all comets have the same composition, or the exact nature of the size distribution of the dust. Astronomers, so far, have recorded about 700 comets out of a suggested population of around a million million in the Solar System. And these 700 represent a very biased selection. The vast majority of the comets known to us happen to be the ones that get to within the orbit of Mars, and even so, only the large ones of that group have been detected.

Only one comet has been visited by spacecraft, and even here, the $16 \times 8 \times 8$ kilometre nucleus was imaged at a resolution of around 200 metres from a closest approach distance of 596 kilometres, while the craft was travelling past at 150,000 miles per hour. So we have a crude idea as to what the nucleus of comet Halley looks like today, but because of its many close perihelion passages, the nucleus has decayed to half its original size, and so we have no idea as to its birthday appearance. Furthermore, comet Halley has an intermediate orbital period, is retrograde and is very much a

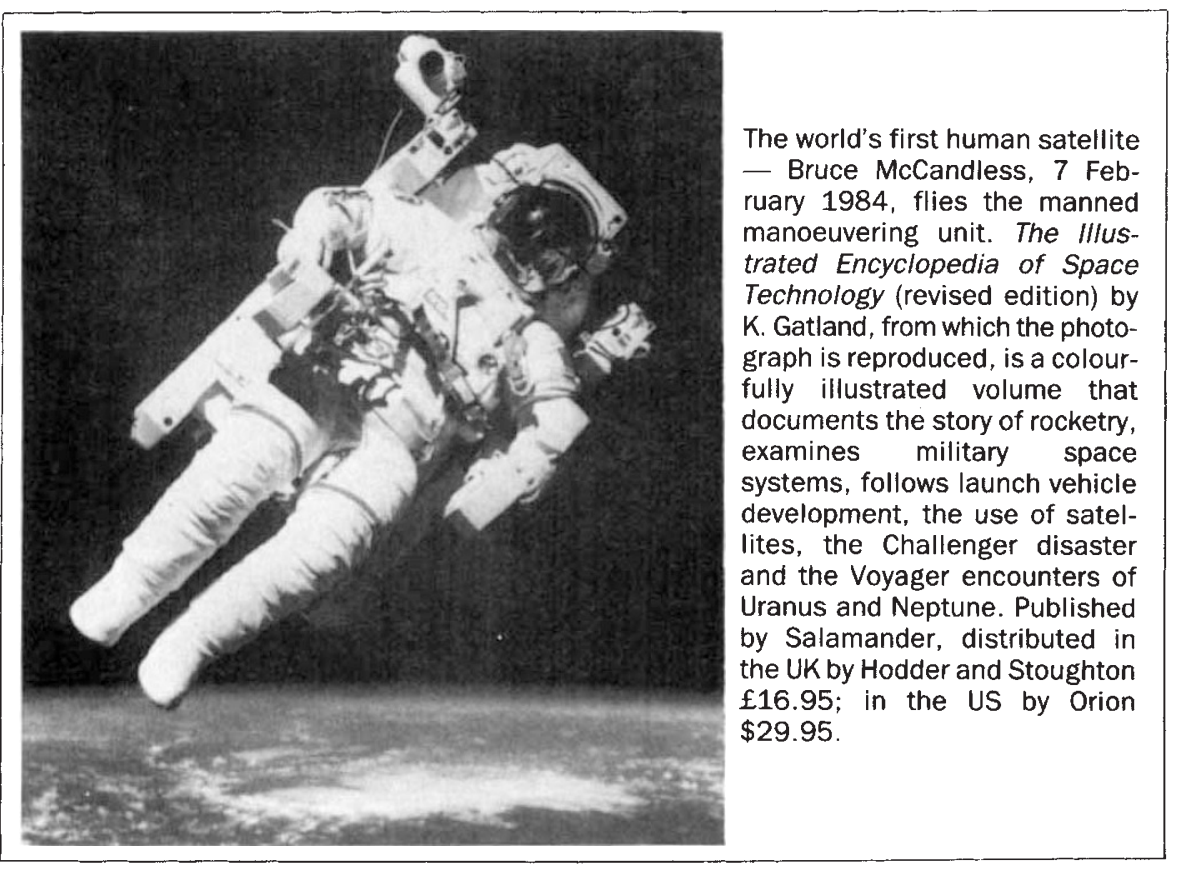

giant, so it could be far from typical. The nucleus has a density that has been estimated to be anywhere between 1.2 and 0.2 grams per $\mathrm{cm}^{3}$. So the relationship between the cometary mass and any observable brightness parameter is rather vague, and the suggestion that the cometary mass distribution is the product of accretion as opposed to fragmentation is subject to debate. The present cometary population has a total mass of the order of that of the Earth, this being a mere shadow of the initial total mass.

Cometary evolution has been very wasteful. Many comets have decayed completely into streams of meteoroid dust, and many have been ejected from the Solar System to wander off around the galactic disk. All stars with planets are thought to have produced comets in profusion, yet we have recorded no obvious hyperbolic comets passing the Sun - we see only the well-mixed remnants of our own cometary cloud and none from other systems. And seeing only a remnant, it is difficult to judge whether this fragment is typical of the original group or whether it had some rather unusual initial orbital characteristics that helped it survive. The subject is also enlivened by the probable existence of an Oort-Öpik belt of comets in a deep-freeze store of radius about a third of the distance to the Sun's stellar neighbours, and a Kuiper cloud of comets in an undetectable refrigerator just beyond the orbit of Pluto.

Bailey, Clube and Napier here provide a magnum opus that seemingly leaves no stone unturned. The reader is treated to an intensive review of mankind's historical notions as to the structure and significance of comets. Much is made of the present-day fashionability of catastrophism, and giant comets, cometary fragmentation and times of high impact flux are hinted at as having occurred in the not too distant past. Detailed analyses are presented of work done in the field of orbital perturbation. The efficacy of Jupiter as the capturer of passing long-period comets is debated in depth. The stability of the Sun's surrounding Oort-Öpik cloud of comets, against the passage of stars and the general galactic tides, that varies in strength as we wobble our way around the galactic nucleus, is investigated.

The Origin of Comets is an authoritative, well-referenced, well-illustrated, comprehensive and profound review of an extremely complex but fascinating minor topic in cosmogony. The fact that we are still not sure where comets came from, or might still come from, does not stop this book being most enjoyable and rewarding. I found it continuously thought-provoking and a spur to further endeavour.

David $W$. Hughes is in the Department of Physics, University of Sheffield, Sheffield S3 $7 R H$, UK.

NATURE $\cdot$ VOL $345 \cdot 21$ JUNE 1990 